\title{
UTILIDAD DE LA ENDOSCOPIA VIRTUAL Y EL URO-TC EN EL DIAGNÓSTICO DE TUMORES DEL TRACTO URINARIO SUPERIOR.
}

Joaquín Ulises Juan Escudero, José Manuel Esteban Hernández', Francisco Sánchez Ballester, Luís de la Torre Abril, Felipe Ordoño Domínguez, Milagros Fabuel Deltoro, Macarena Ramos de Campos, Emilio López Alcina, Pedro Navalón Verdejo y Julio Zaragoza Orts.

Servicio de Urología y ERESA Servicio de Radiodiagnóstico'. Consorcio Hospital General Universitario de Valencia. Valencia. España.

Resumen.- OBJETIVO: Los tumores del tracto urinario superior (TTUS) conforman una patología de incidencia relativamente baja que a menudo suponen un reto diagnóstico para el urólogo ya que, suelen ser necesarias varias pruebas de imagen para alcanzar el diagnóstico definitivo. En los últimos años la tomografía computerizada ha alcanzado un notable desarrollo, y gracias a la aparición de nuevo hardware y software de procesamiento de imágenes actualmente es posible detectar lesiones uroteliales de pequeño tamaño, así como realizar reconstrucciones tridimensionales del tracto urinario y navegación endoscópica virtual por el interior del mismo.
Joaquín Ulises Juan Escudero

Avenida Juan Carlos I, 7

03370 Redován. Alicante. (España)

chimojuan@hotmail.com

Trabajo recibido: 10 de marzo 2006.
MÉTODOS: Entre enero del 2004 y junio de 2005 hemos realizado un total de 15 exploraciones a 15 pacientes durante el estudio de hematuria asintomática de probable origen en el tracto urinario superior, todas ellas indicadas por el Servicio de Urología de nuestro hospital. La téenica incluyó tres fases en la adquisición de imágenes: fase basal, fase vascular y fase excretora. El procesado y análisis de las imágenes fue realizado por un solo radiólogo.

RESULTADOS: Un total de 10 tumores fueron diagnosticados, obteniendo en todos los casos confirmación anatomopatológica. No registramos ninguna complicación derivada de la propia técnica, y todos los pacientes toleraron bien la exploración.

CONCLUSIÓN: EI Uro-TC es una prueba segura, bien tolerada, con una elevada sensibilidad y eficiencia, muy útil en el diagnóstico de la patología renal y del TUS, susceptible de ser utilizada en el futuro como única prueba de imagen radiológica en el estudio de hematuria asintomática y como prueba de elección en el estudio de patología tumoral urológica.

Palabras clave: Tomografía computerizada (TC). Tumores urológicos. Endoscopia virtual.

Summary.- OBJECTIVES: Upper urinary tract (UUT) tumors are a relatively low incidence pathology which often represents a diagnostic challenge for the urologist, because several imaging tests are usually necessary to achieve the final diagnosis. Over the last years CT scan has suffered a notable development and nowadays, with the arrival of new image processing hardware and software, it is possible to detect small urothelial lesions and to perform 3-D urinary tract reconstruction and virtual endoscopic navigation. 
METHODS: Between January 2004 and June 2005 we performed a total of 15 examinations in 15 patients for the study of asymptomatic hematuria of probable upper urinary tract origin. The technique included three phases to obtain images: basal phase, vascular phase and excretory phase. Processing and image analysis was performed by one radiologist.

RESULTS: A total of 10 tumors were diagnosed, with pathological confirmation in all cases. We did not register any complication secondary to the technique, and all patients tolerated well the exam.

CONCLUSIONS: The CT urography is a safe, welltolerated, highly sensitive and efficient imaging test, very useful in the diagnosis of renal and UUT pathology. It may become the only imaging test performed in the study of asymptomatic hematuria and the test of choice for the study of urological tumoral pathology.

Keywords: CT scan. Urological tumors. Virtual endoscopy.

\section{INTRODUCCIÓN}

La frecuencia de los tumores uroteliales del tracto superior ha experimentado un notable aumento en los últimos años a pesar de tratarse de una patología con una baja incidencia. Aunque los tumores de pelvis renal representen sólo un pequeño porcentaje de todas las neoplasias del tracto urinario, constituyendo un $5 \%$ de todos los tumores uroteliales, su incidencia máxima se cifra en 10 casos por 100.000 habitantes y año. Los tumores ureterales son aún menos frecuentes y su incidencia es de 3 a 4 veces menor que los de la pelvis renal. Pero el progresivo envejecimiento de la población parece estar contribuyendo al aumento en la incidencia de estas neoplasias. Este tipo de tumores rara vez se manifiestan antes de los 40 años y la edad media en el momento del diagnóstico es de 65 años. El signo más frecuente de presentación es la hematuria macroscópica o microscópica que se presenta en más del $75 \%$ de los casos, seguido del dolor lumbar (1).

Los pacientes afectos de esta patología urológica, o en los que se sospecha, son sometidos a varias exploraciones, tales como la ecografía, la urografía intravenosa y el TAC o la RM antes de llegar a un diagnóstico etiológico certero de la misma, esto supone una gran incomodidad para el paciente y un gasto excesivo de tiempo y recursos.
La urografía intravenosa (UIV) y la ecografía, que hasta ahora han sido las pruebas de elección para valorar en un primer instante el tracto urinario superior, tienen bastantes limitaciones, como son la baja sensibilidad, sobre todo para lesiones de pequeño tamaño, su baja especificidad a la hora de dilucidar la naturaleza de defectos de repleción intraluminales y el hecho de ser explorador- dependientes.

Desde que Hounsfield y Ambrose publicaran sus primeros resultados en el uso de la tomografía computerizada en 1972 (cuya creación le valió el Nobel de Medicina en 1979), esta técnica ha evolucionado ostensiblemente hasta la actualidad. En un primer momento, la TAC realizaba un solo corte tomográfico en cada vuelta, y siempre en planos axiales. Posteriormente se desarrolló el TAC helicoidal que ha conseguido disminuir la distancia entre cortes aumentado así la definición y la capacidad de distinguir estructuras de menor tamaño. Actualmente disponemos de tomógrafos con múltiples detectores (como son los de 64 detectores, recientemente adquirido en nuestro centro) que permiten obtener hasta 64 cortes en cada vuelta del tomógrafo de $1 \mathrm{~mm}$ aproximado de espesor cada uno, y que no sólo obtienen imágenes en planos axiales, si no que permiten obtener cortes en diferentes planos espaciales. Al realizar varios cortes en una sola vuelta, es posible abarcar una extensión corporal mayor con un menor tiempo de exploración, siendo suficiente con unos 10 segundos para completar una adquisición del cuerpo al completo.

Con la aparición de la tomografía computerizada (TC) multicorte y el desarrollo de los nuevos softwares para el procesamiento de imagen, el urólogo, en un trabajo conjunto con el radiólogo, tiene la posibilidad de obtener información funcional y anatómica del tracto urinario, así como de realizar un estudio endoscópico dinámico. $Y$, todo esto, en un tiempo de adquisición bastante reducido. Así nace la endoscopia virtual, que consiste en una navegación a través del interior del tracto urinario que se realiza tras la reconstrucción tridimensional de los cortes obtenidos mediante el Uro-TC.

La TC ya ha demostrado ser la prueba con mayor sensibilidad y especificidad en la detección y caracterización de masas renales en diferentes estudios $(1,2)$, de hecho está comprobada su mayor eficacia cuando se trata de la evaluación de masas renales, o en el estudio de hematuria microscópica con elevada sospecha de neoformación renal; superando a la ecografía, que ha quedado en muchos casos relegada a un segundo plano, o para ser utilizada en el seguimiento de estas; $y$, por supuesto, a la urografía intravenosa (UIV), que en muchas ocasiones es ineficaz para la detección de dichas masas renales (1). 
La principal ventaja hasta el momento de la UIV ha sido su capacidad de diagnóstico para los defectos de repleción intraluminales en los sistemas colector y excretor, pero con la aparición de las secuencias urográficas bi y tridimensionales del UroTC, que además ofrece imágenes con representaciones mucho mas familiares para los urólogos, ya que muestran la anatomía de la vía excretora tal y como lo hace la UIV, aunque con una mejor definición, parece que ésta puede quedar relegada a un segundo plano.

Por otra parte, si en el protocolo diagnóstico de tumores uroteliales del tracto superior vamos a incluir la TC como estudio de imagen radiológica de extensión, nos planteamos hasta que punto podemos prescindir de la urografía en el algoritmo diagnóstico de esta patología.

\section{MATERIAL Y MÉTODOS}

En el periodo comprendido entre enero del 2004 y junio del 2005 hemos realizado un total de 15 exploraciones a 15 pacientes de los que 7 eran varones y 8 mujeres; con edades comprendidas entre los 57 y los 84 años (media 69 años). La exploración no requería preparación previa, salvo ayunos de 8 horas.

Todos los pacientes fueron sometidos en serie a las siguientes exploraciones: ecografía urológica, UIV y Uro-TC. Todas las exploraciones fueron indicadas por un urólogo de nuestro servicio. En todos los casos la exploración se realizó en el curso del estudio de hematuria asintomática o ante elevada sospecha de neoformación de vías altas. La reconstrucción tridimensional fue llevada a cabo en todos los estudios, completándola con la navegación virtual en cinco de ellos.

Los criterios de exclusión a la hora de realizar la exploración, que son en realidad contraindicaciones relativas a la ejecución de la prueba; fueron: la alergia al contraste iodado y la insuficiencia renal moderada-severa, considerando ésta cuando los niveles de creatinina sérica se encontraban por encima de $2.5 \mathrm{mg} / \mathrm{mL}$. En este último caso, si el paciente necesita ser dializado por su fallo renal, se puede realizar la prueba concertando previamente una sesión de hemodiálisis inmediatamente posterior a la exploración. La única contraindicación absoluta fue el embarazo.

Para llevar a cabo el estudio, se realizaron tres adquisiciones en todos los casos, utilizando un tomógrafo de General Electrics ${ }^{\circledR}$, Lightspeed de 16 detectores; (esto significa que en cada vuelta del tomógrafo se realizan un total de 16 cortes al mismo tiempo, de un espesor de cerca de $0.5 \mathrm{~mm}$ ):

1.- Adquisición en vacío; que se realiza siempre, independientemente de la patología a estudio y de las características del paciente. (Esta, si se realiza como adquisición única, se ha demostrado de gran utilidad en el diagnóstico de litiasis y nos da información sobre la morfología renal y de las estructuras adyacentes).

2.- Fase vascular; que se realiza inmediatamente después de la inyección del material de contraste que muestra la vascularización (arterial o venosa) y la morfología y funcionalidad renal. Lo que pretendemos es por una parte valorar si se encuentra intacta la función renal y, por otra, investigar la presencia de masas renales por la captación de contraste, distinta a la del parénquima renal sano (Figura1). Además en esta fase se puede valorar si existe captación de contraste a cualquier otro nivel del tracto urinario de modo anómalo, lo cual haría sospechar la existencia de un proceso neoformativo incluso antes de que ocurra la opacificación de la vía excretora. Esto es de especial importancia durante el estudio de hematuria macro o microscópica asintomática o elevada sospecha de tumor renal o del tracto urinario.

3.-Fase excretora; por último realizamos una tercera adquisición a los 6-8 minutos de la inyección del contraste, la fase urográfica, en la que se produce la

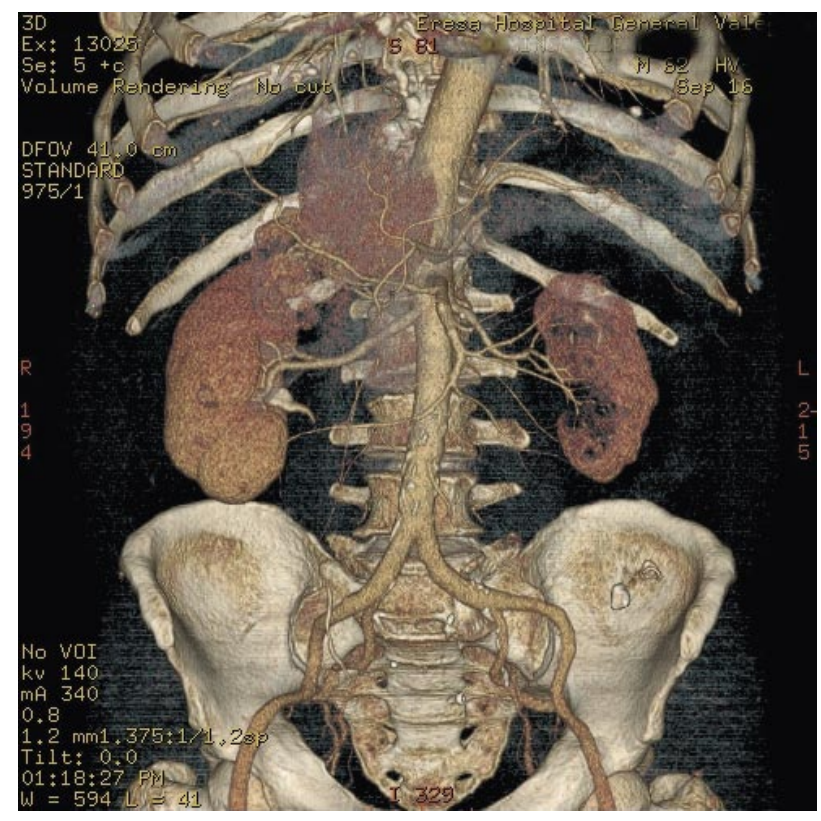

FIGURA 1. Imagen de la adquisición en fase vascular. 
opacificación de la vía excretora (cuando la funcionalidad de la unidad renal está conservada) y nos permite valorar su morfología. Es de especial interés para detectar defectos de repleción intraluminales y es la que nos da la imagen más parecida a la urografía clásica (Figura 2) con la que estamos más familiarizados. En los casos en los que la opacificación no fue satisfactoria se realizó una nueva adquisición en decúbito prono o tras forzar la diuresis.

Para opacificar la vía urinaria se utilizó un medio de contraste iodado no iónico, la lopramida (Clarograf $(\mathbb{R}$ ), inyectándose entre 80 y $100 \mathrm{cc}$ de contraste a una velocidad de $2,5 \mathrm{cc}$ por segundo, con el paciente en decúbito supino, por norma general, y sobre la mesa de exploración utilizando para ello un inyector mecánico.

El procesado de imágenes se realizó en una

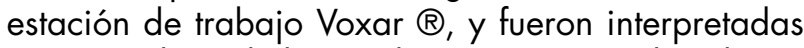
por un solo radiólogo, el mismo que realizó la reconstrucción tridimensional y la navegación virtual en los casos en los que ésta se llevó a cabo.

Para calcular los valores que definen la validez interna de la prueba se ha usado como patrón de referencia, la anatomía patológica. En todos los casos se ha utilizado el esquema clásico de tabla de contingencia, en la que hemos cruzado el resultado

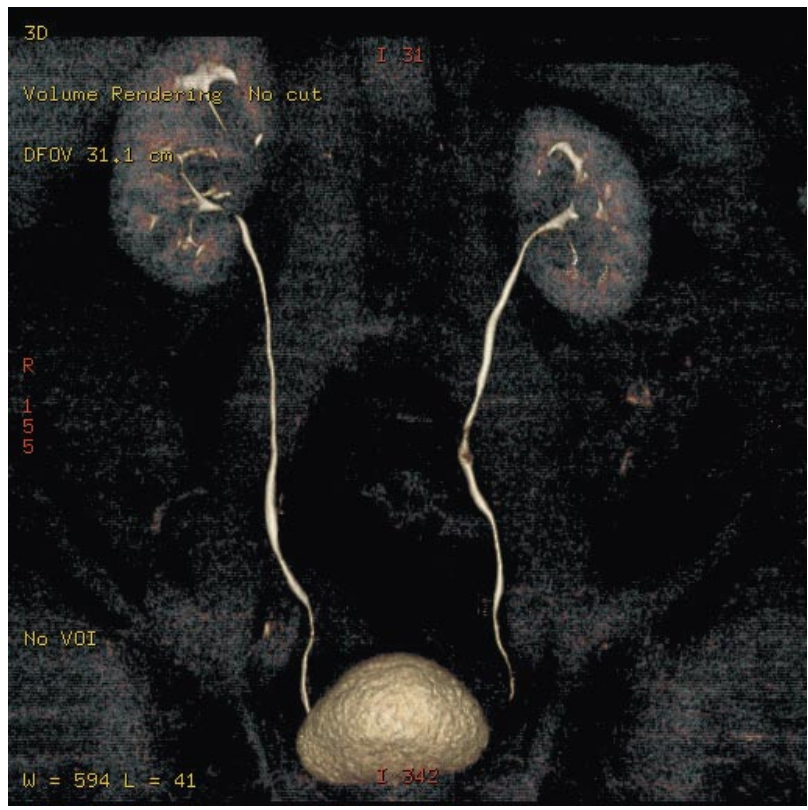

FIGURA 2. Imagen tridimensional de la urografía excretora obtenida mediante Uro-TC. de nuestra prueba con los valores de verdad (estándar de oro) anteriormente mencionados. Las pruebas diagnósticas han sido realizadas en todos los pacientes en serie (por motivos éticos y de optimización de recursos), motivo por el cual en la mayoría de ellos no se realizó la Uro-RNM.

\section{RESULTADOS}

El tiempo invertido en realizar las adquisiciones varió entre 8 y 30 minutos con una media de 12 minutos y para el tratamiento de imágenes se utilizó entre 10 y 35 minutos (media de 17 minutos).

En todos los pacientes se alcanzó el diagnóstico, encontrando 10 neoformaciones, 1 quiste complicado, un traumatismo renal y tres estudios fueron compatibles con la normalidad.

De las 10 neoformaciones: 6 se ubicaban en pelvis renal (Figura 3), 4 eran ureterales (en una se evidenció en el Uro-TC neoformación sincrónica en uréter terminal y en otra de ellas tumor vesical sincrónico). En todos los casos con sospecha de neoformación de vías en el Uro-TC, los pacientes fueron sometidos a intervención, realizándose nefroureterectomía radical el los casos de neoformación del tracto urinario superior (TTUS) y RTU vesical en los casos de

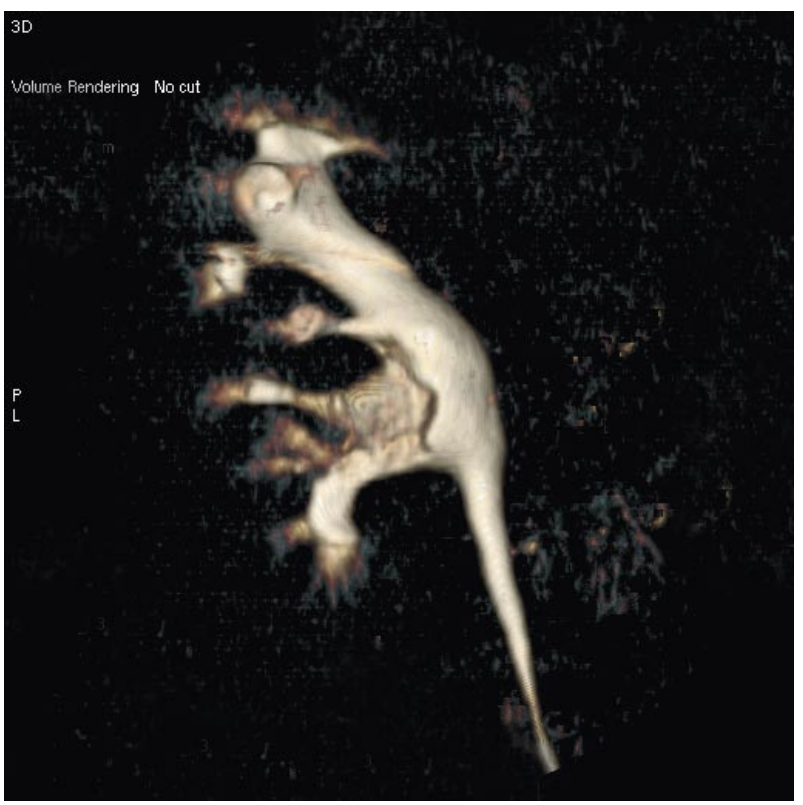

FIGURA 3. Imagen de la adquisición en fase urográfica en la que se aprecia un defecto de replección intraluminal en pelvis renal que correspondió a un tumor uroterial. 
neoformación vesical obteniendo en todos ellos confirmación anatomopatológica del diagnóstico ofrecido previamente por el Uro-TC.

En los restantes casos de hematuria, la causa, confirmada en TC fue: traumatismo renal, que en un primer momento pasó desapercibido y quiste renal complicacado (Bosniak II). En los tres últimos casos la exploración fue compatible con la normalidad, tratándose uno de ellos de un paciente monorreno y portador de una ureterostomía cutánea y catéter ureteral y en tratamiento con dicumarínicos, por lo que se atribuyó a estos la causa de la hematuria. Así mismo sucedió con los restantes dos pacientes, en los que la hematuria fue atribuida a la anticoagulación. En todos los casos la actitud tomada fue conservadora y la evolución de los pacientes favorable.

No hemos registrado reacciones adversas a la administración de contraste y la tolerancia de la prueba fue buena en todos los pacientes. No encontramos variaciones significativas en los niveles de creatinina sérica medidos a las 24 horas de la exploración tras la realización del Uro-CT.

La ecografía reno-vesical previa resultó negativa en 14 de los casos, así pues, solamente consiguió

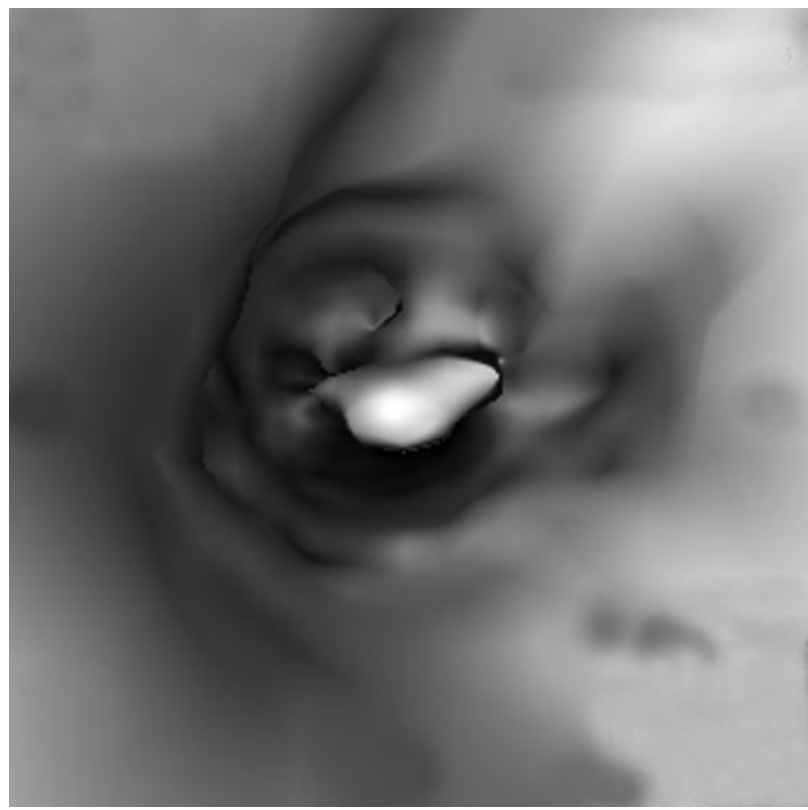

FIGURA 4. Imagen de la endoscopia virtual tras la reconstrucción tridimensional en la que se aprecia la protusión de la neoformación urotelial en el interior del ureter. detectar el caso de neoformación a nivel vesical, describiendo la dilatación subsiguiente a neoformación ureteral en un caso sin poder dilucidar la causa.

En todos los pacientes con sospecha de neoformación por hematuria asintomática se realizó UIV, no evidenciando defectos de repleción en tres de ellos que si fueron diagnosticados con el Uro-CT. En dos de estos casos se realizó RNM, resultando una positiva y una negativa siendo, por lo tanto el único falso negativo.

En el diagnóstico de lesiones tumorales a lo largo del estudio de hematuria asintomática, en nuestra serie y tomando como patrón de referencia la anatomía patológica, la prueba ha mostrado una sensibilidad del $100 \%$, una especificidad del $100 \%$, valor predictivo positivo del $100 \%$ y el valor predictivo negativo ha sido del $100 \%$.

\section{DISCUSIÓN}

EI CT ya forma parte de diferentes protocolos diagnósticos de patología urológica, pero debido a su creciente desarrollo no existe consenso sobre en qué lugar del algoritmo diagnóstico de la patología del trato urinario superior ubicarlo actualmente.

Son varias las publicaciones que han descrito su utilidad en el diagnóstico de patología urológica como la litiasis, los cruces vasculares a nivel de la unión pieloureteral, las anomalías congénitas, las neoplasias primarias del tracto urinario, la valoración de las metástasis y la extensión por contigüidad de las neoplasias urinarias así como para la evaluación de las masas renales $(2,6)$.

En la UIV entre el 50 y el $75 \%$ de los tumores del TUS se presentan como defectos de repleción, cifra ampliamente superada en nuestra serie por el Uro-TC, en el que la sensibilidad alcanzó el $100 \%$ en nuestra serie. De esta forma una neoformación que no protuyese en el interior de la luz pielo-ureteral, (como sucede en el caso de CIS) no se visualizaría en la secuencia urográfica del Uro-TC pero tampoco en la UIV.

Así mismo en otras publicaciones se han descrito protocolos de inclusión del Uro-TC en el algoritmo diagnóstico de la hematuria microscópica asintomática, resaltando la importancia que adquiere en la optimización de recursos la inclusión de esta prueba en el estudio del paciente con hematuria microscópica, sobre todo en los mayores de 40 años en los que está considera como primera prueba de imagen radiológica a realizar (3). 
La urografía ha mostrado en nuestro estudio una sensibilidad y especificidad del $62.5 \%$ y $100 \%$ respectivamente, un valor predictivo positivo del $100 \%$ y un valor predictivo negativo del $57 \%$ para el diagnóstico de neoformación del TUS. Estos valores son ostensiblemente menores que los obtenidos con el Uro-TC cuando éste se realizó para el estudio de hematuria asintomática de posible origen en el TUS.

La sensibilidad que alcanza la resonancia magnética para el diagnóstico de neoplasias del TUS es del $88 \%$ cuando se trata de lesiones mayores de $6 \mathrm{~mm}$ pero cae ostensiblemente cuando se trata de lesiones de menor tamaño (9). La TC de 16 detectores detecta lesiones de hasta $4 \mathrm{~mm}$ pudiendo mejorar el rendimiento con los nuevos aparatos de 64 detectores de los que ya disponen algunos centros de nuestro país. No consideramos válidos los parámetros de validez interna de la RM en nuestro estudio por no haberla realizado de forma sistemática en todos los pacientes.

A la hora de calcular la validez externa de la prueba no se ha tenido en cuenta la prevalencia de la patología tumoral que, como hemos apuntado anteriormente, es baja, por lo que los valores predictivos positivos y negativos de esta exploración obtenidos con nuestro estudio han de ser interpretados con cautela.

En nuestro estudio resulta evidente la utilidad del Uro-TC, ya que no sólo sirvió para el diagnóstico etiológico de la hematuria macroscópica asintomática, sino que podría haber evitado realizar la ecografía y la urografía, ya que en cualquier caso es necesario realizar la TC para el diagnóstico de extensión previo a la cirugía. Así nos permite valorar con un solo estudio el estado del tumor primario, la invasión de órganos vecinos así como su estadío y la extensión a distancia.

Otra ventaja que nos ofrece el Uro-TC es la posibilidad de reconstrucción tridimensional $y$, tras esto, la opción de realizar navegación virtual a través del interior del tracto urinario (Figura 4). Esto no sustituiría en ningún caso a la endoscopia clásica: ni a la cistoscopia ni a la ureterorrenoscopia, pero puede ser una alternativa en casos seleccionados, como son pacientes con elevado riego anestésico - mala tolerancia a la exploración. Pensamos que en caso de sospecha de hematuria o patología de origen vesical y debido a la accesibilidad y relativa sencillez de la cistoscopia, la utilidad clínica del Uro-TC es cuanto menos cuestionable, mientras que cuando se trata de valorar el tracto urinario superior la eficiencia de esta prueba es bastante elevada. La ureteropieloscopia tiene una precisión diagnóstica que alcanza el $86 \%$ para los tumores de pelvis renal y el $90 \%$ para tumores ureterales (1). Ofrece la ventaja respecto a la TC de diagnosticar el carcinoma in situ y en contra el hecho de no ser siempre accesible toda la vía urinaria y tratarse en ocasiones de pacientes con importante comorbilidad asociada, en los que someterse a esta exploración supondría un riesgo adicional, tanto anestésico como derivado de la exploración como, es la perforación ureteral con la consiguiente extravasación de células tumorales.

El problema que más frecuentemente hemos encontrado a la hora de realizar la exploración ha sido los artefactos provocados por los movimientos respiratorios del paciente, este hecho se ve disminuido cuando usamos CT helicoidales multicorte. En nuestro estudio hemos utilizado un CT de 16 detectores, aunque actualmente disponemos de uno de 64 que minimiza el efecto de los movimientos respiratorios sobre la calidad de imágenes (2).

Otro problema que hemos encontrado ha sido la pobre opacificación de la vía excretora en riñones que tienen afectada su función. Para conseguir una mejor visualización de la vía en estos casos ha ayudado, los cambios posturales por un lado y el hecho de forzar la diuresis. Finalmente, otro inconveniente aparece cuando el paciente es portador de clips quirúrgicos o prótesis metálicas de otro tipo, entonces se produce un artefacto alrededor de éstas que dificulta la valoración de las estructuras adyacentes.

Respecto a la dosis recibida de radiación no existe consenso en la literatura, pero muchas publicaciones apuntan a que la radiación recibida es entre 1.5 y 2 veces mayor con la TC que con la urografía (4). Así la dosis efectiva calculada para un TC simple en el estudio de patología litiásica es de 10-15 m Sv, y la del Uro-TC es aproximadamente de 25-35 mSv, frente a los $10 \mathrm{mSv}$ aproximados estimados de dosis efectiva recibida tras la realización de una urografía intravenosa (6). De cualquier forma se están desarrollando protocolos en los que se reduce la radiación a costa de perder calidad de imagen, pero que en casos de litiasis por ejemplo demuestran ser igualmente efectivos.

El coste en nuestro centro de una urografía es de 118,62 euros, mientras que el del Uro-TC ronda los 152,56 euros (menor que el de la RNM que ronda los 283 euros), a pesar de resultar más caro el último hay que tener en cuenta que cuando se realiza la UIV como primera prueba de imagen radiológica, es necesaria una segunda prueba en alrededor de la mitad de los casos, mientras que cuando la primera prueba de imagen es el Uro-TC tan solo el 10\% de 
pacientes requieren exploraciones complementarias adicionales (5). No disponemos de datos sobre el costo exacto de la ecografía urológica en nuestro centro, pero su costo se puede estimar en unos 40 a 60 euros, debido no tanto al de la exploración como por el personal necesario para realizarla.

En el caso de hematuria asintomática, todos los pacientes son sometidos a ecografía, cistoscopia, y UIV. Aunque algunos autores proponen el Uro-TC con cistoscopia virtual como alternativa a la endoscopia vesical clásica $(7,8)$, nosotros pensamos que ésta es necesaria, si bien, si resulta negativa, el paciente se podría ahorrar tanto la ecografía como la urografía, más aún, teniendo en cuenta el alto número de falsos negativos que hemos tenido en nuestra serie y que los verdaderos positivos han requerido la realización de un TC de confirmación y estadiaje. Además, el Uro-TC nos permite realizar la reconstrucción tridimensional y la navegación por el tracto urinario superior (10), incluso cuando la dilatación del mismo es mínima. Esto nunca va a sustituir a la ureterorrenoscopia, que nos permite biopsiar y obtener diagnóstico de certeza. Pero puede ser de gran utilidad en los casos en los que esta no esté indicada o se trate de pacientes de alto riesgo. Además esta navegación es útil a la hora de planificar una actitud terapéutica invasiva.

Por otra parte la realización de una UIV requiere de una hora de tiempo aproximadamente, mientras que para realizar un Uro-TC invertimos tan solo entre 6 y 8 minutos para realizar las 3 adquisiciones necesarias para completar el estudio. Y el procesado de imágenes, cuando es realizado por un radiólogo experimentado no lleva mas de 20 minutos. Además de no precisar preparación intestinal.

\section{CONCLUSIONES}

A pesar de que nuestra serie es pequeña, así como las revisadas en la literatura existente, el Uro-TC en nuestra opinión es una prueba eficiente, segura y cómoda para el paciente, que puede estar al alcance de la mayoría de centros en los que se disponga de la tecnología necesaria.

En nuestra experiencia es indudable su gran utilidad especialmente cuando ha sido realizada durante el estudio de hematuria macroscópica asintomática donde la ecografía y/o cistoscopia no han sido concluyentes.
Además permite en casos seleccionados optimizar recursos sanitarios y agilizar el diagnóstico de la patología urológica. Es previsible que en un futuro se constituya como la prueba de diagnóstico por imagen radiológica de elección fundamentalmente indicado en el estudio de hematuria asintomática.

\section{BIBLIOGRAFÍA y LECTURAS RECOMENDADAS ( ${ }^{*}$ lectura de interés $y^{* *}$ lectura fundamental)}

1. WALSH, P.C.; RETIK, A.B.; VAUGHAN, E.D. Campbell. Urology, 4: 3113, 2005.

**2. KIM, J.K.; CHO, K.S.: "Pictorial review: CT urography and virtual endoscopy: promising imaging modalities for urinary tract evaluation". Br. J. Radiol., 76: 199, 2003.

*3. MAHER, M.; KALRA, M.; RIZZO, S. y cols.: "Multidetector CT Urography in imaging of the urinary tract in patiens with hematuria". Korean Journal of radiology, 5: 1, 2004.

4. NAWFEL, R.D.; JUDY, P.F.; SCHLEIPMAN, A.R. y cols.: "Patient radiation dose al CT urography and conventional urography". Radiology, 232: 126, 2004.

5. GUPTA, K.B.; SILVERMAN, S.G.; McTAVISH, J.D. y cols.: "The impact on diagnosis yield, practice patterns, and cost of using CT urography rather than intravenous urography in the evaluation of hematuria". Radiology, 221: 50, 2001.

**6. CAOILI, E.M.; COHAN, R.H.; KOROBKIN, M. y cols.: "Urinary tract abnormalities: Initial experience with multi-detector row CT urography". Radiology, 222: 353, 2002.

*7. KIM, J.K.; PARK, S.Y.; KIM, H.S. y cols.: "Comparison of virtual cystoscopy, multiplanar reformation, and source CT images with contrast material-filled bladder for detecting lesions". AJR., 185: 689, 2005.

8. BROWNE, R.F.; MURPHY, S.M.; GRAINGER, R. y cols.: "CT cystography and virtual cystoscopy in the assessment of new and recurrent bladder neoplasms". Eur. J. Radiol., 53: 147, 2005.

9. BERNHARDT, T.M.; SCMIDL, H.; PHILIPP, C. y cols.: "Diagnostic potential of virtual cistoscopy of the bladder: MRI vs CT. Preliminary report". Eur. Radiol., 13: 305, 2003.

**10. PRANDO, A.: "CT-virtual endoscopy of the urinary tract". Int. Braz. J. Urol., 28: 317, 2002. 